\title{
Análisis de la percepción de uso de las redes sociales como herramienta de marketing en las MiPYMES de Tamaulipas, México.
}

\author{
Karina Abigail Aldape Nolascoํㅜ, Demian Abrego Almazán², José Melchor Medina Quintero³. \\ abi_1904@hotmail.com,dabrego@uat.edu.mx,jmedinaq@uat.edu.mx. \\ ${ }^{1}$ Maestra en Dirección Empresarial, Universidad Autónoma de Tamaulipas, Tamaulipas, 8710o, Victoria, México. \\ ${ }^{2}$ Profesor-Investigador de la Universidad Autónoma de Tamaulipas, Tamaulipas, 8710o, Victoria, México. \\ ${ }^{3}$ Profesor-Investigador de la Universidad Autónoma de Tamaulipas, Tamaulipas, 8710o, Victoria, México.
}

DOI: 10.17013/risti.18.49-65

Resumen: Un negocio debe estar donde se encuentren sus clientes potenciales, por esta razón las organizaciones están innovando para mantenerse al alcance de los mismos, una forma es a través de las redes sociales. El propósito de ésta investigación es determinar el grado de aceptación tecnológica de Facebook en actividades de marketing a través del Modelo de Aceptación Tecnología en las micro, pequeñas y medianas empresas (MiPYMES) de Tamaulipas México. Para alcanzarlo, se utiliza el modelado de ecuaciones estructurales con la aplicación de 129 cuestionarios. La evidencia empírica muestra resultados positivos y significativos en la mayoría de las variables estudiadas, también sugiere la falta de una cultura en cuanto al uso, manejo y aceptación de Facebook como herramienta de marketing en la mayoría de las empresas consultadas. Se espera que los resultados sean punto de partida para investigadores en este ámbito de estudio.

Palabras-clave: Facebook; Modelo de Aceptación Tecnología; Mínimos Cuadrados Parciales; Marketing.

\section{Analysis of the perception of the use of social media as a marketing tool in MSMEs of Tamaulipas, Mexico.}

\begin{abstract}
A business should be where its potential customers are. Therefore, organizations are always looking for ways to keep themselves as close to their customers as possible. One way to achieve that is through the social media. The goal of this study is to determine Facebook's degree of technological acceptance in marketing activities undertaken by micro, small and medium-sized enterprises' (MSMEs) of the state of Tamaulipas Mexico through the technological acceptance model (TAM). To achieve that goal, a structural equation model is used with the administration of 129 questionnaires. The empirical evidence shows positive and significant outcomes in most of the researched variables. The evidence also suggests the lack of a culture where Facebook can be used, managed and accepted as a marketing tool in most of the participating enterprises. It is argued that the results of this study can be used as a starting point for researchers addressing this topic.
\end{abstract}

Keywords: Facebook; Technology Acceptance Model; Partial Least Squares; Marketing. 


\section{Introducción}

La presencia de millones de usuarios en sitios de medios sociales es característicamente atractivo para las empresas, ya que abre nuevos canales para interactuar con los consumidores y otras partes interesadas importantes, como proveedores y empleados, por esta razón, los diferentes tipos de organizaciones de todo el mundo están tratando de integrar los medios sociales en sus diversos aspectos de procesos de negocio y operaciones (Fogel, 2010). Asimismo Fonseca (2014) expone que las redes sociales son la evolución de las tradicionales maneras de comunicación del ser humano, que han avanzado con el uso de nuevos canales al convertirse en una herramienta la cual se basa en la creación, conocimiento colectivo y confianza generalizada.

La Asociación Mexicana de Internet (AMIPCI) en su estudio de Marketing Digital y Redes Sociales (2013) resalta que las principales actividades de los mexicanos en Internet son el uso de las redes sociales, las cuales ocupan el primer lugar, mientras que los datos estadísticos de la empresa Owloo (2014) muestra que México cuenta con 56 millones de usuarios en Facebook, lo cual representa un área de oportunidad para las empresas debido al gran número de clientes potenciales (Kaplan y Haenlein, 2010; Gálvez, 2013).

Sin embargo, y a pesar de los beneficios y usos que las redes sociales pueden ofrecer a las organizaciones, hay muchos directivos que no han decidido adentrarse en este tipo de tecnología (AMIPCI, 2013), no obstante en un corto plazo las empresas tendrán que adoptar e incorporarlas de manera estratégica a su organización (Coté y Vecina, 2005; Turban y Volonino, 2011). Por ello, el propósito de la presente investigación es determinar el grado de aceptación tecnológica de Facebook en las MiPYMES, de la zona centro del estado de Tamaulipas, México, a través del Modelo de Aceptación Tecnología propuesto por Davis en 1989.

Para alcanzarlo se realizó una revisión de la literatura, así como, la aplicación de una encuesta a 129 MiPYMES, los datos obtenidos fueron analizados mediante la técnica de Mínimos Cuadrados Parciales (PLS, Partial Least Squares, por sus siglas en inglés). Por último, el presente documento se ha estructurado de la siguiente forma: una sección para la revisión de la literatura y otra para la descripción del método utilizado y del modelo propuesto; seguido, del análisis de los resultados, y finalmente en el último apartado se muestran las principales aportaciones al conocimiento obtenidas, dentro de los cuales destaca una limitada cultura en cuanto al uso, manejo y aceptación de las redes sociales como herramienta de marketing en la mayoría de las unidades económicas investigadas.

\section{Revisión de la literatura 2.1. Redes sociales}

Las redes sociales están transformando la participación y el compromiso de los consumidores (Liang y Turban, 2011), por ello, las empresas se han enfocado en aprovechar sus ventajas, ya que, la generalización del uso de Internet y sus herramientas ha supuesto la llegada de un canal de comunicación que permite poner en contacto a una gran cantidad de personas día tras día (De Ugarte, 2006). Cada vez con mayor frecuencia los individuos utilizan formatos online con el fin de relacionarse y compartir experiencias (Kozinets, 2002). Algunos autores contemplan el uso de las redes sociales 
como uno de los elementos de cambio en la forma de competir de las empresas del mundo globalizado, y que su análisis puede constituir una buena herramienta para la obtención de información relevante (Gálvez y Martín, 2009).

Por otra parte, las redes sociales forman parte del marketing digital ya que ofrecen un diálogo entre los participantes, este tipo de canales permiten difundir servicios, productos o una marca en forma directa, y masiva a prospectos o clientes (Vela, 2014; Akdoğan y Altuntaş, 2015), los cuales a su vez la difunden a su red de contactos entendiéndose por Social Media Marketing a las acciones de Marketing aplicadas a las redes sociales (Blogs, Comunidades online como Facebook, Twitter, Foros, Wikis, entre otras), con el objetivo de dar a conocer y compartir información o contenidos para aumentar la popularidad, prestigio y visibilidad de una empresa o marca (Kaplan y Haenlein, 2010). Estos ambientes tienen la ventaja de permitir una comunicación directa con los clientes sin la necesidad de realizar una gran inversión (Belo et al., 2013), por lo tanto, ayudan a disminuir costos de marketing, (Kozinets, 2002; Ellison, 2007); reducen los costos de servicios técnicos (Dholakia y Vianello, 2006), o incluso favorecen la creación de lazos afectivos y fortalecen los niveles de lealtad hacia el producto, marca u organización (Koh y Kim, 2004). Así, crear una campaña de marketing efectiva en redes sociales permite ahorrar en publicidad, como también generar un tipo de publicidad más agradable y mejor recibida por los consumidores, ya que se logra establecer una relación más cercana con ellos, generando finalmente lealtad de marca. Para lograr buenos resultados, es importante que las empresas interactúen a diario en las redes sociales, y mostrar un interés en participar de estas plataformas de comunicación, como también en cultivar la relación con los clientes (Lee et al., 2012; Chaffey et al., 2012).

De acuerdo a un estudio realizado por la AMIPCI (2013), menciona que la red social con mayor presencia de marcas es Facebook, también este estudio refleja que el 59\% de los que siguen a alguna marca dentro de las redes sociales se ha visto influenciado en sus decisiones de compra. Fonseca (2014) afirma que las redes sociales mejoran oportunidades de negocio, rentabilidad y permanencia en el mercado para las empresas, ya que les genera un mayor acercamiento a los consumidores y clientes potenciales, trabajar en red con personas y empresas afines, hacer participar a sus clientes en el desarrollo de su negocio y posicionarse como referente en su sector.

\subsection{Modelo de aceptación de la tecnología}

Actualmente, el uso óptimo de las tecnologías de la información dentro de las organizaciones es una necesidad, ante su influencia en la producción de bienes y servicios. Esto, aunado a que estas tecnologías son cada vez más asequibles en el mercado, resaltando entonces su gestión adecuada, en lugar de la inversión realizada (Moon y Kim, 2001; Lin y Sher, 2007). Por ello, en las últimas décadas, diversas líneas de investigación se han centrado en identificar determinados factores de inferencia en los comportamientos de aceptación y uso de cierto tipo de tecnología por parte de los usuarios finales (Yong et al., 2010; Sigg et al., 2014).

Lo anterior dio como resultado la existencia de diversos modelos y teorías para medir la aceptación de la tecnología; entre los que destaca el Modelo de Aceptación de la Tecnología (TAM, Technology Acceptance Model) introducido por Davis en 1989 
(Mathieson, 1991; Venkatesh y Davis, 2000; Sigg et al., 2014), debido a que es un modelo efectivo altamente probado en predecir el uso de las tecnologías de información (Yong et al., 2010). El TAM tiene sus raíces en la Teoría de la Acción Razonada (TRA) de Ajzen y Fishbein (1980), pero reemplaza varias de las medidas de actitud del modelo de la Acción Razonada por medidas de aceptación tecnológica con el fin de predecir la conducta, aceptación e intención de uso de las tecnologías por parte de los individuos (Davis et al., 1989; Wu, 2005; Huh et al., 2009). Sin embargo, al limitarse al campo de los sistemas y tecnologías de la información, reducen el número de variables de control aplicables con respecto a TRA (Taylor y Todd, 1995; Blas et al., 2008).

El modelo trata de predecir la aceptación tecnológica basado en dos variables principales (Gefen et al., 2003; Yong et al., 2010): La utilidad percibida - grado en que una persona cree que el uso de un determinado sistema mejora su rendimiento en el trabajo-; y en la facilidad de uso percibida - grado en que una persona cree que utilizando un sistema en particular, podrá liberarse del esfuerzo que le conlleva realizar un trabajo -.

Es decir, el modelo sugiere que cuando a los usuarios se les presenta una nueva tecnología, una serie de factores influyen en su decisión sobre cómo y cuándo la van a utilizar. Según Davis et al. (1989), el propósito primario del TAM es explicar las causas de aceptación de las tecnologías por los usuarios. Por lo tanto, el modelo propone que las percepciones de un individuo en la utilidad percibida y la facilidad de uso percibida de una tecnología, sean concluyentes para determinar su intención de usarla.

Por otra parte, la revisión de la literatura permitió detectar la falta de estudios relacionados con las redes sociales y TAM en México, sin embargo se detectó que en otras latitudes existen, pero en una limitada cantidad. Una de estas investigaciones examinó la adopción y uso de las redes sociales virtuales por parte de los usuarios incluyendo en el modelo aspectos como la confianza y el riesgo percibido hacia las mismas, los resultados de dicho estudio apoyan una relación e influencia positivas entre las variables (Romero et al., 2011). Asimismo, otro estudio realizado en España y Portugal se efectuó con el objetivo de identificar las variables que influyen en la intención de usar esta Red Social para sus decisiones de compra. Los resultados de este estudio indican que la intención de uso de Facebook en las decisiones de compra está influenciada por la utilidad percibida, la influencia social y la actitud. Además, este estudio identifica que el disfrute percibido podría tener un papel esencial, incluso superior a la utilidad percibida, en la determinación de la actitud del individuo hacia Facebook como herramienta para la búsqueda de información sobre el producto a comprar (Miranda et al., 2014).

\section{Método}

El presente estudio plantea un modelo conceptual, en el que se propone que la actitud de uso se ve afectada por la facilidad de utilidad y por la utilidad percibida, siendo esta última inferida también por la facilidad de utilidad. De la misma manera, se plantea que tanto la utilidad percibida como la actitud de uso son antecedentes directos de la intención de uso, la cual a su vez influye en la percepción del uso actual de cierta tecnología, de este último constructo y para efecto de la presente investigación se mide a través del grado de aceptación tecnológica de la herramienta tecnológica denominada Facebook. La figura 1, muestra el modelo a probar y sus relaciones causales propuestas. 


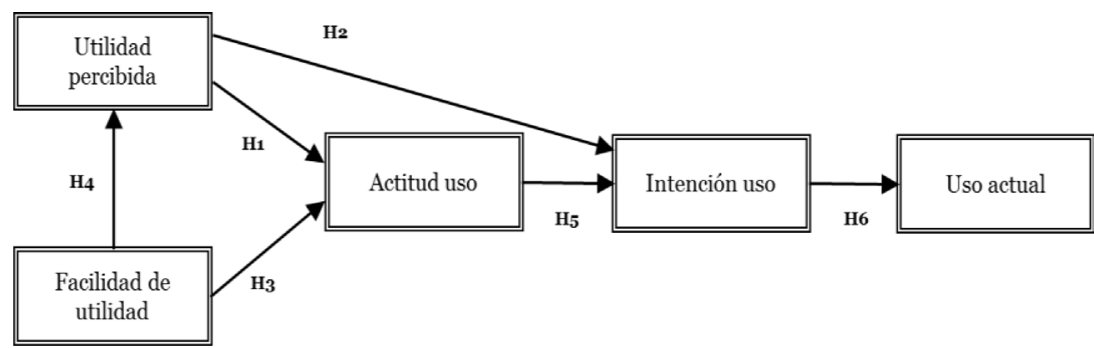

Figura 1 - Modelo teórico a probar

Por otra parte, las suposiciones a probar del modelo de investigación propuesto se resumen en la Tabla 1, en la cual además del fundamento teórico suministrado por el modelo de Davis de 1989, se anexan referencias complementarias que apoyan las hipótesis planteadas.

\begin{tabular}{|c|c|c|}
\hline Variable & Hipótesis & Referencias \\
\hline \multirow{2}{*}{$\begin{array}{l}\text { Utilidad } \\
\text { percibida }\end{array}$} & $\begin{array}{l}\text { H1. La percepción de utilidad que tienen las } \\
\text { PYMES respecto a la Red Social Facebook tiene } \\
\text { una influencia positiva en la actitud de uso de las } \\
\text { redes sociales como herramienta de marketing. }\end{array}$ & \multirow{2}{*}{$\begin{array}{l}\text { Davis et al. (1989); Venkatesh y Davis } \\
\text { (2000); Huang et al. (2007); Willis } \\
\text { (2008); Venkatesh y Bala (2008); } \\
\text { Huh et al. (2009); Yang y Lin (2011); } \\
\text { Chen et al. (2011); Lorenzo et al. } \\
\text { (2011); Chen y Chen (2011); Casaló et } \\
\text { al. (2012); Miranda et al. (2014). }\end{array}$} \\
\hline & $\begin{array}{l}\text { H2. La percepción de utilidad que tienen las } \\
\text { MiPYMES respecto a la Red Social Facebook } \\
\text { influye de manera positiva en la intención de } \\
\text { uso de las redes sociales como herramienta de } \\
\text { marketing. }\end{array}$ & \\
\hline \multirow{2}{*}{$\begin{array}{l}\text { Facilidad de } \\
\text { uso }\end{array}$} & $\begin{array}{l}\text { H3. La facilidad de uso percibida de Facebook } \\
\text { como herramienta de marketing tiene un } \\
\text { efecto positivo en la actitud de uso en las } \\
\text { MiPYMES. }\end{array}$ & \multirow{2}{*}{$\begin{array}{l}\text { Davis (1989); Davis et al., (1989); } \\
\text { Venkatesh y Morris, (2000); } \\
\text { Venkatesh et al. (2003); Park (2010); } \\
\text { Chung et al. (2010); Lorenzo et al. } \\
\text { (2011); Chen y Chen (2011); Casaló et } \\
\text { al. (2012); Miranda et al. (2014). }\end{array}$} \\
\hline & $\begin{array}{l}\text { H4. El efecto que tiene la facilidad de uso de } \\
\text { Facebook percibida por las MiPYMES es positivo } \\
\text { en la percepción de utilidad. }\end{array}$ & \\
\hline $\begin{array}{l}\text { Actitud de } \\
\text { uso }\end{array}$ & $\begin{array}{l}\text { H5. La actitud de los empresarios afecta } \\
\text { positivamente en la intención de uso de Facebook } \\
\text { como herramienta de marketing. }\end{array}$ & $\begin{array}{l}\text { Davis et al. (1989); Bernadette } \\
\text { (1996); Van Der Heijden (2003); } \\
\text { Bhattacherjee y Premkumar (2004); } \\
\text { Shin y Kim (2008); Lorenzo et al. } \\
\text { (2011); Chen y Chen (2011). }\end{array}$ \\
\hline $\begin{array}{l}\text { Intención de } \\
\text { uso }\end{array}$ & $\begin{array}{l}\text { H6. La intención de uso de Facebook como } \\
\text { herramienta de marketing en las MiPYMES influye } \\
\text { de forma positiva en su uso actual de esta Red } \\
\text { Social. }\end{array}$ & $\begin{array}{l}\text { Davis (1989); Taylor y Todd (1995); } \\
\text { Henderson y Divett (2003); Wu } \\
\text { y Wang (2005); Venkatesh y Bala } \\
\text { (2008); Chen et al. (2011); Lorenzo et } \\
\text { al. (2011). }\end{array}$ \\
\hline
\end{tabular}

Tabla 1 - Resumen de hipótesis

En relación con el proceso de creación del instrumento, éste se fundamentó en la revisión de la literatura, lo cual permitió detectar distintos modelos de operacionalización de las variables relacionadas con el tema, que han sido utilizado satisfactoriamente en estudios 
anteriores, por lo que el primer boceto del instrumento fue enviado para su revisión a un grupo de investigadores y profesionales del área. De cada experto se recibió su opinión sobre la congruencia, relevancia, suficiencia y claridad de los ítems-factores propuestos; paso seguido, se realizó una prueba piloto, todo con el fin de obtener un instrumento fácil de comprender e interpretar, el cuestionario resultante está conformado por 29 ítems, de los cuales 8 son de respuesta múltiple y 21 de escala tipo Likert de 5 puntos (1. Totalmente en desacuerdo a 5. Totalmente de acuerdo).

Para la obtención de la muestra, se partió de los registros proporcionados por el Sistema de Información Empresarial Mexicano (SIEM, http://www.siem.gob.mx) en donde para agosto de 2015, se tenían registradas 2,851 MiPYMES, las cuales se encuentran dentro de la ubicación geográfica establecida (Tamaulipas, México) para la investigación. Después de identificarlas, se gestionó la autorización correspondiente con las diferentes Cámaras y Asociaciones de Comercio y Servicio de las localidades seleccionadas para el apoyo en la aplicación del instrumento. El trabajo de campo se realizó a través de un muestreo a conveniencia entre los meses de septiembre a diciembre del 2015, a través de visitas in situ y explicando al sujeto de investigación el objetivo del estudio, en este caso el informante clave abordado fueron los gerentes generales y de marketing, debido a que reúnen el perfil deseado, la muestra final lograda fue de 129 MiPYMES de los diferentes sectores económicos que conforman a la zona de estudio seleccionada.

\section{Resultados}

Después de aplicar y contabilizar la información, los resultados revelan que la muestra se integra por empresas del sector comercio con un 69,14\% y de servicios con 30,86\%, esto coincide con los datos del censo económico de unidades económicas realizado por el INEGI (2014) donde señala que en el estado de Tamaulipas en el sector privado, las empresas comerciales representan la mayoría de las unidades económicas. Asimismo el 49\% de las organizaciones tienen de 0-10 trabajadores, de 11-30 trabajadores lo representa el 30\% y el 21\% de 31-100 empleados; igualmente se les solicitó a los sujetos de investigación que indicaran el puesto que desempeñan dentro de la organización, obteniendo que el mayor porcentaje de las personas encuestadas son los dueños con un $44 \%$, seguido de los gerentes generales representado por un $27 \%$ y jefes del departamento de ventas con un $29 \%$.

Por otra parte, para comprobar el modelo, se aplicó el modelado de ecuaciones estructurales (MES) mediante la técnica estadística denominada PLS, a través del paquete informático SmartPLS versión 3. Esta técnica exige evaluar la calidad del modelo antes de obtener su validación estructural (Ringle et al., 2014). Por lo tanto, se realizaron pruebas de los principales criterios de calidad, comenzando por el análisis de la fiabilidad individual del ítem, el cual establece que para aceptar un indicador como integrante de un constructo reflectivo, este debe poseer una carga factorial $(\lambda)$ o correlaciones simples iguales o superiores a 0,707 (Carmines y Zeller, 1979). Tomando como referencia el criterio de aceptación anterior $(\lambda \geq 0,707)$, se eliminaron los indicadores FU3 y FU4. La Tabla 2 muestra los resultados. 


\begin{tabular}{cccccccccccc}
\hline \multicolumn{3}{c}{ Actitud de uso $(\boldsymbol{A C})$} & \multicolumn{4}{c}{ Intención de uso (INT) } & \multicolumn{3}{c}{ Uso actual (UA) } \\
\hline Ítem & $\lambda$ & & T-Statistic & Ítem & $\lambda$ & T-Statistic & Ítem & $\lambda$ & \multirow{T}{*}{ T-Statistic } \\
\hline$A C 1$ & 0,815 & $* * *$ & 17,502 & $I N T 1$ & 0,847 & $* * *$ & 23,386 & $U A 1$ & 0,935 & $* * *$ & 53,078 \\
\hline$A C 2$ & 0,913 & $* * *$ & 43,856 & $I N T 2$ & 0,928 & $* * *$ & 68,891 & $U A 2$ & 0,959 & $* * *$ & 110,033 \\
\hline$A C 3$ & 0,913 & $* * *$ & 38,959 & $I N T 3$ & 0,889 & $* * *$ & 28,648 & $U A 3$ & 0,753 & $* * *$ & 9,090 \\
\hline$A C 4$ & 0,914 & $* * *$ & 49,938 & $I N T 4$ & 0,922 & $* * *$ & 69,522 & & & & \\
\hline
\end{tabular}

\begin{tabular}{lccccccc}
\hline & \multicolumn{3}{c}{ Facilidad de uso $(F U)$} & \multicolumn{4}{c}{ Utilidad percibida (UP) } \\
\hline Ítem & $\lambda$ & & T-Statistic & Ítem & $\lambda$ & T-Statistic \\
\hline$F U 1$ & 0,746 & $* * *$ & 9,940 & $U P 1$ & 0,814 & $* * *$ & 18,902 \\
\hline$F U 2$ & 0,784 & $* * *$ & 14,909 & $U P 2$ & 0,906 & $* * *$ & 46,145 \\
\hline$F U_{3}$ & 0,593 & $* * *$ & 7,548 & $U P 3$ & 0,879 & $* * *$ & 27,809 \\
\hline$F U 4$ & 0,611 & $* * *$ & 6,173 & $U P 4$ & 0,906 & $* * *$ & 35,707 \\
\hline$F U 5$ & 0,821 & $* * *$ & 16,903 & $U P 5$ & 0,887 & $* * *$ & 36,150 \\
\hline$F U 6$ & 0,778 & $* * *$ & 12,754 & $U P 6$ & 0,888 & $* * *$ & 38,407 \\
\hline
\end{tabular}

*** Valor $\mathrm{t}>3,310(\mathrm{p}<\mathrm{0}, 001),{ }^{* * *}$ valor $\mathrm{t}>2,586(\mathrm{p}<\mathrm{o}, 01),{ }^{*}$ valor $\mathrm{t}>1,965(\mathrm{p}<0,05)$

Tabla 2 - Fiabilidad Individual

Continuado con las pruebas de calidad del modelo, la siguiente fase es determinar la fiabilidad del constructo, la cual se evalúa mediante el Alfa de Cronbach ( $\alpha$ ) y el Coeficiente de fiabilidad compuesta ( $\rho c)$, que en ambos casos su interpretación es similar. Por lo cual se utiliza las directrices ofrecidas por Chin (1998) quien sugiere 0,7 como punto de referencia. La Tabla 3 muestra los resultados y como se observa, todos los constructos son fiables y poseen una consistencia interna satisfactoria.

\begin{tabular}{lcc}
\hline Constructos & Alfa Cronbach $(\boldsymbol{\alpha})$ & Fiabilidad Compuesta (pc) \\
\hline Utilidad percibida & 0,942 & 0,949 \\
\hline Facilidad de uso & 0,807 & 0,865 \\
\hline Intención de uso & 0,919 & 0,943 \\
\hline Actitud de uso & 0,911 & 0,938 \\
\hline Uso actual & 0,874 & 0,916 \\
\hline
\end{tabular}

Tabla 3 - Fiabilidad del constructo

Por otra parte, también se debe calcular el promedio de la varianza extraída (AVE, por sus siglas en inglés). Este coeficiente indica la cantidad de varianza que un constructo reflectivo obtiene de sus indicadores con relación a la cantidad de varianza debido al 
error de medida y su valor debe ser mayor que 0,5 (Fornell y Larcker, 1981) y como se puede observar en la Tabla 4 todas las medidas AVE son válidas.

\begin{tabular}{ll}
\hline Constructos & AVE \\
\hline Utilidad percibida & 0,775 \\
\hline Facilidad de uso & 0,565 \\
\hline Actitud de uso & 0,792 \\
\hline Intención de uso & 0,805 \\
\hline Uso actual & 0,787 \\
\hline
\end{tabular}

Tabla 4 - Validez convergente

Por último, se analizaron los valores de la matriz de correlaciones entre constructos, formada por la raíz cuadrada del coeficiente AVE obtenido de cada uno, señalando que dichos valores deben ser superiores al resto de su misma columna (Chin, 1998). Como se puede observar en la Tabla 5 los constructos cumplen con dicho criterio.

\begin{tabular}{llllll}
\hline Constructos & $\begin{array}{l}\text { Actitud } \\
\text { de uso }\end{array}$ & $\begin{array}{l}\text { Facilidad } \\
\text { de uso }\end{array}$ & $\begin{array}{l}\text { Intención } \\
\text { de uso }\end{array}$ & $\begin{array}{l}\text { Uso } \\
\text { actual }\end{array}$ & $\begin{array}{l}\text { Utilidad } \\
\text { percibida }\end{array}$ \\
\hline Actitud de uso & $\mathbf{0 , 8 9 0}$ & & & & \\
\hline Facilidad de uso & 0,438 & $\mathbf{0 , 7 5 1}$ & & & \\
\hline Intención de uso & 0,848 & 0,396 & $\mathbf{o , 8 9 7}$ & & \\
\hline Uso actual & 0,363 & 0,350 & 0,423 & $\mathbf{0 , 8 8 7}$ & \\
\hline Utilidad percibida & 0,801 & 0,481 & 0,768 & 0,444 & $\mathbf{0 , 8 8 0}$ \\
\hline
\end{tabular}

Tabla 5 - Validez Discriminante

Una vez que se ha podido comprobar que los constructos son fiables y válidos, se procedió a calcular el peso y la magnitud de las relaciones entre las distintas variables, lo anterior mediante la utilización de dos índices propuestos por Johnson et al. (2006): i) La varianza explicada $\left(\mathrm{R}^{2}\right)$ - la cual permite determinar el poder predictivo del modelo, por ello sus valores deben ser iguales o mayores a 0,1 ya que menores proporcionan poca información (Falk y Miller, 1992); y ii) los coeficientes path estandarizados ( $\beta$ ), los cuales muestran la fuerza de las relaciones entre las variables dependientes e independientes, por lo cual sus valores deben de alcanzar al menos un o,2 para que se consideren significativos (Chin, 2000) y como se observa en la Tabla 6 los valores obtenidos para $\mathrm{R}^{2}$ están dentro de rangos convenientes.

\begin{tabular}{ll}
\hline Constructos & Varianza explicada \\
\hline Utilidad percibida & 0,232 \\
\hline Actitud de uso & 0,645 \\
\hline Intención de uso & 0,741 \\
\hline Uso actual & 0,179 \\
\hline
\end{tabular}

Tabla 6 - Varianza Explicada $\left(\mathrm{R}^{2}\right)$ 
Cabe señalar que se recurrió a la técnica no paramétrica Bootstrap, con un procedimiento de remuestreo con reemplazo, considerando 129 casos con 5000 muestras, lo cual es recomendado para resultados finales (Hair et al., 2014), de lo anterior se obtuvieron los valores $t$ de student y la significancia $(p)$. Para una distribución $t$ de Student de dos colas con $n$ grados de libertad, siendo $n$ el número de muestras a considerar en la técnica Bootstrap, los valores que determinan la significancia estadística son: t $(95 \%)=1,965$ ${ }^{*}, \mathrm{t}(99 \%)=2,586 * *$, y t $(99,9 \%)=3,310 * * *$. Como se observa en la Tabla 7 , solo la hipótesis planteada $\mathrm{H}_{3}$, no resulta significativa.

\begin{tabular}{|c|c|c|c|c|c|}
\hline & Hipótesis & & Coeficiente ( $\beta)$ & t Values & p Values \\
\hline$H 1$ & Utilidad percibida $\rightarrow$ Actitud de uso & + & 0,768 & 13.618 & $O, O O O$ \\
\hline $\mathrm{H} 2$ & Utilidad percibida $\rightarrow$ Intención de uso & + & 0,247 & 2.941 & 0,003 \\
\hline$H_{3}$ & Facilidad de uso $\rightarrow$ Actitud de uso & + & 0,069 & 1.160 & 0,246 \\
\hline $\mathrm{H}_{4}$ & Facilidad de uso $\rightarrow$ Utilidad percibida & + & 0,481 & 6.223 & $O, O O O$ \\
\hline$H_{5}$ & Actitud de uso $\rightarrow$ Intención de uso & + & 0,650 & 8.037 & $O, O O O$ \\
\hline H6 & Intención de uso $\rightarrow$ Uso actual & + & 0,423 & 7.272 & $O, O O O$ \\
\hline
\end{tabular}

Tabla 7 - Resultados del modelo estructural.

Por otra parte, para medir la bondad predictiva de los constructos dependientes del modelo se ha recurrido al procedimiento al test Stone-Geisser o parámetro $\mathrm{Q}^{2}$ (Cross Validated Redundancy). Esta prueba se calcula por medio de la técnica blindfolding. Cabe señalar que el valor de este parámetro debe ser mayor a cero para que el constructo tenga validez predictiva (Sellin, 1995; Chin, 1998). Como se observa en la Tabla 8, todos los valores $\mathrm{Q}^{2}$ están por encima de cero, lo cual apoya la relevancia predictiva del modelo en relación con las variables latentes endógenas.

\begin{tabular}{ll}
\hline Constructos & $\mathbf{Q}^{\mathbf{2}}$ \\
\hline Utilidad percibida & 0,175 \\
\hline Actitud de uso & 0,502 \\
\hline Intención de uso & 0,592 \\
\hline Uso actual & 0,119 \\
\hline
\end{tabular}

Tabla 8 - Stone-Geisser Test $\left(\mathrm{Q}^{2}\right)$

Por último, se calculó el valor del Residual Estandarizado de la Raíz Cuadrada Media ( $S R M R$, por sus siglas en inglés), el cual se interpreta como la diferencia promedio entre las correlaciones (varianzas y covarianzas) pronosticadas y observadas, basada en el error estándar del residual, por lo tanto, se puede considerar como una medida de bondad de ajuste para modelos PLS-SEM (Henseler et al., 2014). Sus valores deben oscilar entre 0,0 (ajuste perfecto) y menores a 0,08 para considerarse como válidos (Hu y Bentler, 1999), en este sentido, el valor de SRMR obtenido para el modelo de investigación propuesto es de 0,069, lo cual indica un adecuado nivel de ajuste. Una vez obtenidos los resultados de la modelización $P L S$, se procede a aceptar los coeficientes 
path y, por extensión confirmar las hipótesis formuladas que resulten significativas. Para ello, la Figura 2 muestra los resultados obtenidos.

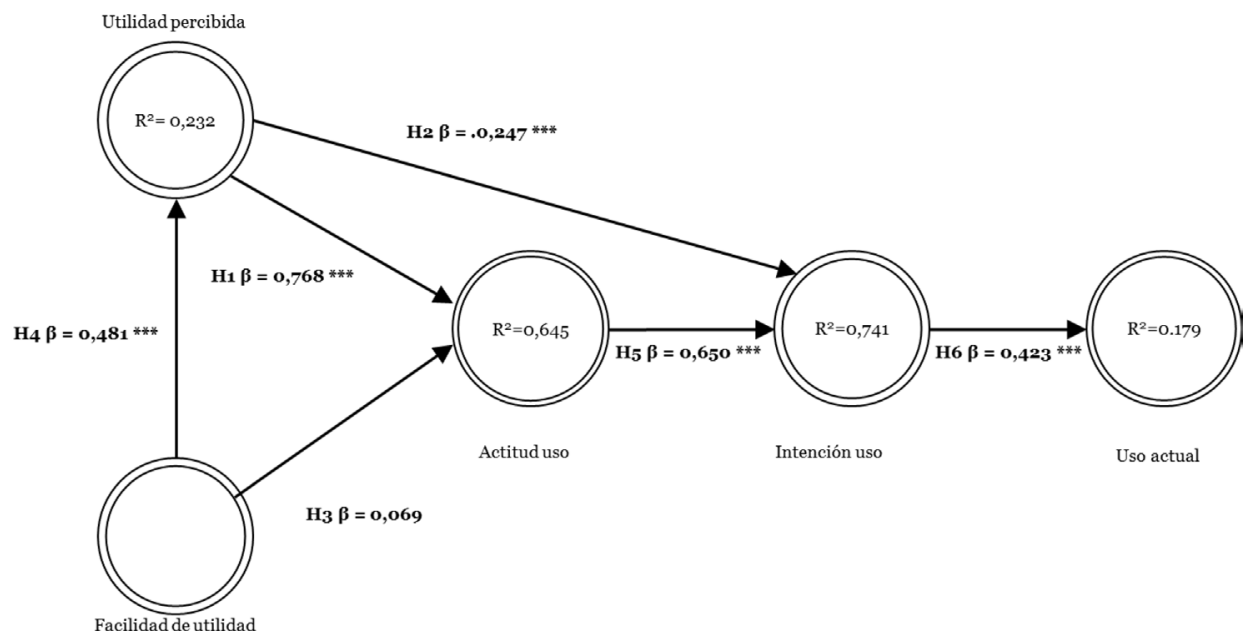

Figura 2 - Coeficientes Path y significancia estadística.

\subsection{Interpretación de los resultados}

De la Figura 2, se puede inferir lo siguiente: que la percepción de utilidad que tienen las MiPYMES respecto a la red social Facebook tiene una influencia positiva y significativa (H1: $\beta=0,768^{* * *}$ ) en la actitud de uso de las redes sociales como herramienta de marketing teniendo una relación causal de forma directa, por lo cual la hipótesis $\mathrm{H} 1$ es aceptada, esto es comparable a la investigación de Huang et al. (2007) en la cual sus resultados indican que la percepción de la utilidad de los usuarios influyen en su actitud de utilizar el aprendizaje electrónico, y además esta afirmación se comprueba en la teoría TRA, que establece que las actitudes hacia una conducta están influidas por las creencias relevantes.

Mientras que en la relación entre utilidad percibida e intensión de uso (Hipótesis H2) es aceptada, debido a que la percepción de utilidad que tienen las MiPYMES respecto a la red social Facebook influye de manera positiva en la intención de uso de las redes sociales como herramienta de marketing $\left(\beta=0,247^{* *}\right)$, lo cual comprueba que cuanto más fácil sea interactuar con una tecnología, mayor es el sentimiento de eficacia del usuario y, por ende, muestra una mayor intención de usarla (Chung, 2005), asimismo son afines con las investigaciones de Miranda et al. (2014) y Huh et al. (2009) ya que en ambas la percepción de utilidad influye significativamente en la intención de utilizar el sistema.

Ahora bien, en cuanto a la facilidad de uso de Facebook y su inferencia en el constructo actitud de uso, como se puede observar no existe una relación significativa en la actitud hacia su empleo en la empresa (H3: $\beta=0,069)$, por lo tanto se rechaza la hipótesis, el resultado encontrando es muy similar a lo obtenido en la investigación de Miranda et al. (2014) en donde también se investigó esta relación en el ámbito de redes sociales 
e igualmente fue rechazada, esto se puede deber a que la mayoría de las personas entrevistadas percibían fácil el manejo como uso personal, sin embargo, en el ámbito empresarial, desconocen en su mayoría las actividades que se pueden realizar lo que se ve directamente reflejado en su actitud en cuanto al uso y manejo de esta red social.

Con respecto a lo planteado en la hipótesis cuatro, se observa un efecto significativo de la facilidad de uso sobre la percepción de utilidad, por lo tanto, esta hipótesis es aceptada demostrando que el efecto que tiene la facilidad de uso de Facebook percibida por las MiPYMES es positivo en la percepción de utilidad $\left(\mathrm{H}_{4}: \beta=0,481\right)$ de esta forma, la facilidad de uso contribuye a la mejora del desempeño de la tarea, lo que supone un ahorro de esfuerzo, gracias a la facilidad de uso, a fin de lograr más y mejores resultados con el mismo esfuerzo (Davis et al., 1992).

Por otra parte, la relación entre actitud de uso e intención de uso fue aceptada (hipótesis $\mathrm{H}_{5}$ ), ya que demuestra que este constructo ejerce una importante influencia sobre la intención de uso de esta red social $\left(\beta=0,65^{* * *}\right)$, al demostrar que la intención se determina por la actitud que el empresario tenga del uso de Facebook, este resultado concuerda con Lorenzo et al. (2011) en el estudio de redes sociales y su uso en España, quien menciona que la actitud hacia las redes sociales virtuales influyen positiva y significativamente sobre la intención de usarlas.

En lo referente con la hipótesis H6, se determinó una relación causal entre la intención de uso y el uso actual de Facebook, obteniendo un efecto positivo y significativo sobre el nivel de uso final $\left(\beta=0,423^{* * *}\right)$, por tanto, se considera que la intención de uso actúa como variable intermediaria entre el efecto ejercido por las percepciones (facilidad de uso y utilidad percibida) y el uso final del individuo, lo cual es similar a lo obtenido en la investigación de Chen et al. (2011) ya que los resultados obtenidos por los autores muestran una correlación positiva y significativa entre los aspectos de intención de uso y el uso actual, y de igual forma son afines con la Romero et al. (2011) debido a que los resultados de dicho estudio apoyan una relación e influencia positivas entre las variables intención de uso y el uso actual.

\section{Conclusiones y limitaciones}

En relación al uso de Facebook en las empresas de comercio y servicio la zona centro del estado de Tamaulipas se encontró que el 56\% ha incorporado esta herramienta para sus actividades mercadológicas mientras que el $44 \%$ no lo utiliza o difícilmente se usa, lo que representa que casi la mitad de la muestra desconoce el uso y por lo tanto los beneficios de estos medios. Así también, a través del procesamiento de datos se ha identificado que únicamente el 3\% de las empresas realizan una planeación estratégica para llevar a cabo sus actividades de marketing, lo que representa un dato alarmante ya que parte de la efectividad en resultados del marketing digital es una planeación estratégica (Fonseca, 2014; Jayaram et al., 2015).

Otro de los resultados obtenidos en relación al uso actual de Facebook en las organizaciones, es el tiempo que éstas invierten para realizar sus publicaciones de publicidad, promociones, ventas y en general sus actividades de marketing específicamente en esta red social, denotándose que la mayoría de las empresas invierten poco tiempo, es de llamar la atención que menos del 10\% de las empresas invierte por lo 
menos media jornada de trabajo diaria en la dedicación a estos medios de comunicación con el cliente, lo que indica una baja cultura de su uso y en general a realizar estrategias para incrementar las ventas organizacionales.

En cuanto a la comprensión de las funciones que Facebook ofrece para las organizaciones sólo 1 de cada 3 empresarios conoce la totalidad de las actividades que se pueden llevar a cabo en esta red social, esto representa una falta de capacitación en los empresarios, lo cual concuerda con Kaplan y Haenlein (2010), quienes mencionan que muchos ejecutivos son incapaces de identificar efectivamente las oportunidades y amenazas relacionadas a las redes sociales, en parte debido a una falta de entendimiento de su estructura y funcionamiento, a su vez puede ser un área de oportunidad para la creación de nuevas empresas en este ramo para capacitar a los futuros y actuales empresarios, así como gerentes y puestos relacionados al área de marketing, así también es un área de oportunidad para incorporar en los programas de educación superior para que los futuros líderes empresariales posean este conocimiento. Por otro lado, en relación a los resultados de utilidad que los empresarios perciben de esta red social, la mayoría se encuentra de acuerdo en que efectivamente dicha herramienta es útil para su organización y para aquellas empresas que no han incorporado Facebook en las instituciones que dirigen, su percepción de utilidad es alta.

Como conclusión general, las investigaciones e indicadores estadísticos muestran que la estrategia de difusión en redes sociales es una excelente herramienta para el posicionamiento de un mercado de las organizaciones debido a que las redes sociales han causado un gran impacto en los consumidores y principalmente las empresas buscan ser reconocidas por éstos siendo una de las razones del negocio posicionarse en la mente del consumidor, asimismo, el conjunto de los beneficios de sus usos estratégicos, traerá como consecuencia una buena imagen corporativa de la empresa ante los consumidores. Así también cabe destacar que, a través de un conjunto de acciones estratégicas como la planeación en el manejo de redes sociales, son las que tendrán un efecto sobre el posicionamiento de una empresa dentro un mercado, logrando así estar a la vanguardia en el uso y aprovechamiento de las redes sociales, optimizar los canales de comunicación Cliente/Organización que por años se han venido estudiando y que a fin de cuentas ya es una realidad en las economías globales.

Por otra parte, esta investigación presenta ciertas limitantes que se deben considerar antes generalizar los resultados, la primera se relaciona con la validación de un modelo, la cual no puede ser establecida sobre la base de un solo estudio, dado que los datos representan una instantánea en el tiempo. En segundo término esta lo relacionado con el ámbito geográfico (zona centro de Tamaulipas, México), por ello, las investigaciones futuras deberían utilizar una muestra más grande y adoptar un enfoque longitudinal, así también se puede incluir y explorar las interrelaciones entre o variables individuales u organizacionales y su efecto sobre el uso de Facebook. Por lo tanto, las críticas a las relaciones causa-efecto detectadas se deben realizar con cautela. 


\section{Referencias}

Akdoğan, M., y Altuntaş, B. (2015). Covert Marketing Strategy and Techniques. ProcediaSocial and Behavioral Sciences, 207, 135-148. DOI:10.1016/j.sbspro.2015.10.162.

Ajzen I., y Fishbein M. (1980). Understanding attitudes and predicting social behavior. Prentice-Hall.

Bernadette (1996). Empirical evaluation of the revised technology acceptance model. Management science, 42(1), 85-92.

Belo, A., Castela, G., y Fernandes, S. (2013). Ambientes Colaborativos Virtuais: potencial das redes sociais. O caso das empresas do Algarve. RISTI-Revista Ibérica de Sistemas e Tecnologias de Informação, 12, 65-79. DOI: 10.4304/risti.12.65-79.

Bhattacherjee, A., y Premkumar, G. (2004). Understanding changes in beliefs and attitude toward Information Technology usage: A theoretical model and longitudinal test. MIS Quarterly 28, 229-254.

Blas, S., Mafé, C., y Manzano, J. (2008). La influencia de la dependencia del medio en el comercio electrónico B2C. Propuesta de un modelo integrador aplicado a la intención de compra futura en Internet. Cuadernos de Economía y Dirección de la Empresa, 11(36), 45-75.

Carmines, G., y Zeller, R. (1979). Reliability and validity assessment (17). Sage publications.

Casaló, L., Flavián, C., y Guinalíu, M. (2012). Redes sociales virtuales desarrolladas por organizaciones empresariales: antecedentes de la intención de participación del consumidor. Cuadernos de Economía y Dirección de la Empresa, 15(1), 42-51.

Côté, L., Vezina, M., y Sabourin, V. (2005). The strategic management process in e-business. Ivey Business Journal, 69(5), 1-7.

Chaffey, D., Smith, P., y Smith, P. (2012). eMarketing eXcellence: Planning and optimizing your digital marketing. Routledge.

Chen, C., y Chen, P. (2011). Applying the TAM to travelers' usage intentions of GPS devices. Expert Systems with Applications, 38(5), 6217-6221. DOI:10.1016/j. eswa.2010.11.047

Chen, M., Lu, T., Chen, K., y Liu, C. (2011). A TAM-based study on senior citizens' digital learning and user behavioral intention toward use of broadband network technology services provided via television. African Journal of Business Management, 5(16), 7099-7110.

Chin, W. (1998). The Partial Least Squares approach to Structural Equation Modelling. En Marcoulides G.A. (Ed.). Modern Methods for Business Research. Mahwah, NJ: Lawrence Erlbaum Associates.

Chin, W. (2000). Partial Least Square for researchers: An overview and presentation of recent advances using the PLS approach, ICIS 2000 Proceedings. 741-742. 
Chung, D. (2005). Something for nothing: understanding purchasing behaviors in social virtual environments. Cyberpsychology \& behavior, 8(6), 538-554. DOI:10.1089/ cpb.2005.8.538.

Chung, J., Park, N., Wang, H., Fulk, J., y McLaughlin, M. (2010). Age differences in perceptions of online community participation among non-users: An extension of the Technology Acceptance Model. Computers in Human Behavior, 26(6), 16741684. DOI:10.1016/j.chb.2010.06.016

Davis, F. (1989). Perceived usefulness, perceived ease of use, and user acceptance of information technology. MIS quarterly, 319-340. DOI: 10.2307/249008

Davis, F., Bagozzi, R., y Warshaw, P. (1989). User acceptance of computer technology: a comparison of two theoretical models. Management science, 35(8), 982-1003.

Davis, F., Bagozzi, R., y Warshaw, P. (1992). Extrinsic and intrinsic motivation to use computers in the workplace1. Journal of applied social psychology, 22(14), 11111132. DOI: $10.1111 / \mathrm{j} .1559-1816.1992 . t b 00945 \cdot \mathrm{x}$

De Ugarte, D. (2006). El poder de las redes. Recuperado el 24 febrero de 2015, de http://www.deugarte.com/gomi/el_poder_de_las_redes.pdf.

Dholakia, U., y Vianello, S. (2006). A study of sharing in consumer communities. In 35th EMAC Conference, Atenas (23-26).

Ellison, N. (2007). Social network sites: Definition, history, and scholarship. Journal of Computer-Mediated Communication, 13(1), 210-230. DOI: 10.1111/j.10836101.2007.00393.

Estadísticas de Facebook (Owloo), Recuperado el 1 de noviembre de 2014, de https://www.owloo.com

Falk, R., y Miller, N. (1992). A Primer for Soft Modeling, the University of Akron Press: Akron.

Fogel, S. (2010). Problemas de la medición de la palabra de la boca en el social media marketing. Diario Internacional de Comunicaciones Integradas de Marketing, 2, 54-60.

Fonseca A. (2014). Marketing Digital en redes sociales: Lo imprescindible en Marketing Online, Calendar, Palma Área, España.

Fornell, C., y Larcker, D. (1981). Evaluating structural equation models with unobservable variables and measurement error. Journal of Marketing Research, 18, 39-50. DOI: $10.2307 / 3151312$

Gálvez I. (2013) - Facebook para empresas, primera edición, Editorial IC. Antequera, Málaga.

Gálvez, P., y Martín, C. (2009). Redes sociales como fuente de capital social: una reflexión sobre la utilidad de los vínculos débiles. RISTI - Revista Ibérica de Sistemas e Tecnologias de Informação, 3, 13-24. 
Gefen, D., Karahanna, E., y Straub, D. (2003). Inexperience and experience with online stores: The importance of TAM and trust. Engineering Management, IEEE Transactions on, 5O(3), 307-321. DOI 10.1109/TEM.2003.817277

Hair, J., Hult, G., Ringle, C., y Sarstedt, M. (2014). A Primer on Partial Least Squares Structural Equation Modeling (PLS-SEM). Thousand Oaks: Sage.

Henderson, R., y Divett, M. (2003). Perceived usefulness, ease of use and electronic supermarket use. International Journal of Human-Computer Studies, 59(3), 383395. DOI: 10.1016/S1071-5819(03)ooo79-X

Henseler, J., Dijkstra, T., Sarstedt, M., Ringle, C., Diamantopoulos, A., Straub, D., Ketchen, D., Hair, J., Hult, G., y Calantone, R. (2014). Common beliefs and reality about partial least squares: Comments on Rönkkö and Evermann. Organizational Research Methods, 17(2), 182-209. DOI: 10.1177/1094428114526928

Huang, J., Lin, Y., y Chuang, S. (2007). Elucidating user behavior of mobile learning: A perspective of the extended technology acceptance model. The Electronic Library, 25(5), 585-598. DOI http://dx.doi.org/10.1108/02640470710829569.

Hu, L., y Bentler, P. (1999). Cutoff criteria for fit indexes in covariance structure analysis: Conventional criteria versus new alternatives. Structural Equation Modeling, 6, 1-55. DOI: 10.1080/10705519909540118

Huh, H., Kim, T., y Law, R. (2009). A comparison of competing theoretical models for understanding acceptance behavior of information systems in upscale hotels. International Journal of Hospitality Management, 28(1), 121-134. DOI:10.1016/j.ijhm.2008.06.004.

Jayaram, D., Manrai, A., y Manrai, L. (2015). Effective use of marketing technology in Eastern Europe: Web analytics, social media, customer analytics, digital campaigns and mobile applications. Journal of Economics, Finance and Administrative Science. DOI: 10.1016/j.jefas.2015.07.001.

Johnson, M., Herrmann, A., y Huber, F. (2006). The evolution of loyalty intentions. Journal of marketing, $70(2), 122-132$. DOI: http://dx.doi.org/10.1509/jmkg.70.2.122.

Kaplan, A., y Haenlein, M. (2010). Users of the world, unite! The challenges and opportunities of social media. Business Horizons, 53, 59-68. DOI: 10.1016/j. bushor.2009.09.003.

Koh J., y Kim, D. (2004). Knowledge sharing in virtual communities: an e-business perspective. Expert systems with applications, 26(2), 155-166. DOI: 10.1016/ So957-4174(03)00116-7.

Kozinets, R. (2002). The field behind the screen: using netnography for marketing research in online communities. Journal of marketing research, 39(1), 61-72. DOI: http://dx.doi.org/10.1509/jmkr.39.1.61.18935 
Lee, W., Xiong, L., y Hu, C. (2012). The effect of Facebook users' arousal and valence on intention to go to the festival: Applying an extension of the technology acceptance model. International Journal of Hospitality Management, 31 (3), 819-827. DOI: 10.1016/j.ijhm.2011.09.018.

Liang, T., y Turban, E. (2011). Introduction to the special issue social commerce: a research framework for social commerce. International Journal of Electronic Commerce, 16(2), 5-14.

Lin, C., y Sher, P. (2007). Integrating technology readiness into technology acceptance: The TRA Model. Psychology y Marketing, 24(7), 641-657. DOI: 10.1002/mar.20177

Lorenzo, C., Gómez, M., y Alarcón, M. (2011). Redes sociales virtuales: ¿de qué depende su uso en España? Innovar, 21(41), 145-158.

Marketing Digital y redes sociales (AMIPCI) Recuperado el 3 de noviembre de 2014, de https://www.amipci.org.mx.

Mathieson, K. (1991). Predicting user intentions: comparing the technology acceptance model with the theory of planned behavior. Informations and stems research, 2(3), 173-191. DOI: http://dx.doi.org/10.1287/isre.2.3.173

Miranda, F., Rubio, S., Chamorro, A., y Loureiro, S. (2014). Determinantes de la intención de uso de Facebook en el proceso de decisión de compra. Investigaciones europeas de dirección y economía de la empresa, 21(1), 26-34. DOI: 10.1016/j. iedee.2014.05.001

Moon,J.,yKim,Y.(2001).ExtendingtheTAMforaWorld-Wide-Webcontext.Information y management, 38(4), 217-230. DOI: 10.1016/So378-7206(00)00061-6.

Park, N. (2010). Adoption and Use of Computer-Based Voice Over Internet Protocol Phone Service: Toward an Integrated Model. Journal of Communication, 6o(1), 40-72. DOI: 10.1111/j.1460-2466.2009.01440.x.

Ringle, C., Wende, S., y Becker, J. (2014). Smartpls 3. Hamburg: SmartPLS. Academy of Management Review, 9, 419-445. Retrieved from http:// www. smartpls.com.

Romero, C., de Amo, M., y Borja, M. (2011). Adopción de redes sociales virtuales: ampliación del modelo de aceptación tecnológica integrando confianza y riesgo percibido. Cuadernos de Economía y Dirección de la Empresa, 14(3), 194-205. DOI:10.1016/j.cede.2010.12.003

Sigg, M., Cisneros, J., Reyes, S., y Salcedo, J. (2014). Explicación de la adopción de tecnologías de información en Pequeñas Empresas usando el Modelo del Usuario Perezoso: un caso de estudio. RISTI-Revista Ibérica de Sistemas e Tecnologias de Informação, E1 (03), 91-104. DOI: 10.4304/risti.e1.91-104.

Sellin, N. (1995). Partial least square modeling in research on educational achievement. En: Wilifred Bos y Rainer H. Lehmann (Eds.). Reflections on Educational Achievement, New York, USA: Waxmann Munster, 256-267. 
Shin, D., y Kim, W. (2008). Applying the technology acceptance model and flow theory to cyworld user behavior: implication of the web2. O-user acceptance. CyberPsychology \& Behavior, 11(3), 378-382.

Taylor, S., y Todd, P. (1995). Decomposition and crossover effects in the theory of planned behavior: A study of consumer adoption intentions. International journal of research in marketing, 12(2), 137-155. DOI: 10.1016/0167-8116(94)ooo19-K.

Turban, E., y Volonino, L. (2011). Information Technology for Management: Improving Strategic and Operational Performance .Hoboken.

Vela, D. (2014). Social Media Manager. Edición española. Ediciones Anaya Multimedia, Grupo Anaya, S.A.

Venkatesh, V., y Bala, H. (2008). Technology acceptance model 3 and a research agenda on interventions. Decision sciences, 39(2), 273-315. DOI: 10.1111/j.15405915.2008.00192.x.

Venkatesh, V., y Davis, F. (2000). A theoretical extension of the technology acceptance model: Four longitudinal field studies. Management science, 46(2), 186-204. DOI: http://dx.doi.org/10.1287/mnsc.46.2.186.11926.

Venkatesh, V., Morris, M., Davis, G., y Davis, F. (2003). User acceptance of information technology: Toward a unified view. MIS quarterly, 425-478.

Willis, T. (2008). An evaluation of the technology acceptance model as a means of understanding online social networking behavior. ProQuest.

Wu, S. (2005). What drives mobile commerce? : An empirical evaluation of the revised technology acceptance model. Information and Management, 42 (2005), 719-729. DOI: 10.1016/j.im.2004.07.001.

Van der Heijden, H. (2003). Factors influencing the usage of websites: the case of a generic portal in The Netherlands. Information and management, 4O(6), 541-549. DOI: 10.1016/So378-7206(02)00079-4.

Yang, S., y Ling, C. (2011). Factors affecting the intention to use Facebook to support problem- bassed learning among employees in a Taiwanese manufacturing company. African Journal of Business Management, 5(22), 9014-9022.

Yong, L., Rivas, L., y Chaparro, J. (2010). Modelo de aceptación tecnológica (TAM): un estudio de la influencia de la cultura nacional y del perfil del usuario en el uso de las TIC. Innovar. Revista de Ciencias Administrativas y Sociales, 20(36), 187-203. 